\title{
Curative effect observation on deformed auricle treated with EarWell Infant Ear Correction System with modular parents' nursing education
}

Original article

Wei Weia, Ren-Zhong Luo ${ }^{a}$, Zhen-Yun Huanga, Rui Mi,*\%

a Department of Otolaryngology, Guangzhou Children and Women's Medical Center, Guangdong, Guangzhou 51000, China

${ }^{b}$ Department of Physical Medicine and Rehabilitation, Guangzhou CanAm International Medical Center, Guangdong, Guangzhou 51000, China

Received: 21 December 2019; Accepted: 3 January 2020; Published: 20 June 2020

Abstract: Objective: To investigate the efficacy and patient satisfaction of the EarWell Infant Ear Correction System combined with modular parents' nursing education's curative effect on deformed auricle.

Methods: A total of 42 patients (29 boys and 13 girls; 73 ears; age $\leq 3$ months) with auricle deformities who had received EarWell Infant Ear Correction System's treatment and modular parents' nursing education in Guangzhou Children and Women's Medical Center between April and October 2018. The modular parents' education program is standardized by EarWell system. Physician and patients' parents compared the severity of auricle deformity separately before and after the treatment by using the auricle deformities visual analogue scales (VAS) rating system. Patient satisfaction was evaluated by using global aesthetic improvement scale (GAIS). The data collected of auricle deformities VAS and GAIS satisfaction score were applied to measure the treatment's effectiveness.

Results: All the 42 patients (73 external ears) completed the treatment with EarWell Infant Ear Correction System and modular parents' nursing education. The mean age at initiation of treatment was $37.87 \pm 19.44$ days and the therapeutic time span was $47.21 \pm 17.36$ days. At the end of treatment, the physician's and patients' guardians rating of the severity of auricle deformity were significantly improved separately compared to the initial rating $(8.33 \pm 1.27$ vs. $6.51 \pm 0.84 ; P<0.005)(5.77 \pm 1.59$ vs. $8.19 \pm 2.38 P<0.05)$. During the treatment and parents' home nursing care period, the side effect and complications were minor like skin eczema and irrigation; there were no severe complications such as necrosis of the skin and cartilage. The patient tolerance for the treatment was acceptable with the adequate parents' nursing care. Most patients' guardians were satisfied with the treatment outcomes of EarWell Infant Ear Correction System with more engagement of nursing care, the GAIS's rating were increased from pretreatment stage's $26.19 \%$ to treatment completed stage's $90.48 \%$, and the difference was statistically significant $(P<0.05)$.

Conclusions: In this study, we proved that EarWell Infant Ear Correction System with its unique parents' modular nursing care education, as a noninvasive treatment, reasonably improved auricle morphological malformation, and patients' guardians satisfaction with few complications, which is worthy of a wildly clinical promotion.

Keywords: EarWell Infant Ear Correction System • modular parents' nursing care education • auricle deformities • VAS rating system • GAIS rating system

(c) Shanxi Medical Periodical Press.

How to cite this article: Wei W, Luo RZ, Huang ZY, Mi R. Curative effect observation on deformed auricle treated with EarWell infant ear correction system with modular parents' nursing education. Front Nurs. 2020; 2: $153-160$.

* Corresponding author.

E-mail: 450149082@qq.com (W. Wei).

2 Open Access. ๑ 2020 Wei et al., published by Sciendo. (c) BY-NC-ND This work is licensed under the Creative Commons Attribution NonCommercial-NoDerivatives 4.0 License. 


\section{Introduction}

Auricle deformities is a very common neonatal ear congenital malformation disease that include morphological and structural abnormalities. Auricle structure abnormalities are caused by embryologic maldevelopment, which are characterized by an abnormal chondrocutaneous component with an abnormally shaped pinna. Auricle morphological abnormalities are characterized by a normal chondrocutaneous component with an abnormalityshaped pinna caused by deforming forces. Children with auricle deformities and early clinical inrventions were missed in proper time window and will experience more psychological stress, self-distrust, behavioral problems, and social avoidance than those with normal auricle shape. ${ }^{1}$

Early intervention is critical for every child with auricle deformities at birth. Recent research indicates that auricle morphological abnormalities can usually be corrected by appropriate nonsurgical splinting in the neonatal period, but majority of structural abnormalities, such as microtia, are treated with auricle reconstruction operation. ${ }^{2}$ Traditional treatment for congenital auricle deformities has been to wait until a child aged 5-6 years to perform surgical correction with general anesthesia. The risk of surgical operation's complications is divided into two types, early complications (hematoma, infection, fistulae caused by stitches and granulomas, allergic reactions, pressure ulcers, feeling of pain, and asymmetry in side comparison) and late complications (recurrences, telephone ear, excessive edge formation, auricle fitting too closely, narrowing of the auditory canal, keloids, and complete collapse of the ear). ${ }^{3}$

Since the late 1980 s, many studies stated that auricle nonsurgical correction should be started immediately after birth (realistically, at latest by the third day after birth) in order to obtain satisfactory and irreversible results. The mechanism is that the external ear is easily pliable during the neonatal period due to the flexibility of the cartilage and permanent correction can be achieved by forcing the ear into the desired position for several weeks by using an ear splint device. The clinical outcome will release patient family's psychological stress and avoid the general anesthesia and surgical correction's complications. ${ }^{4}$ This article presents our experience with EarWell Infant Ear Correction System splint therapy and modular parents' nursing care education for babies with auricle deformities, from the perspective of pediatric otolaryngologist. Since the adequate and modularized parents' nursing care education may improve the clinical outcome by decreasing the complication such as scratching ears, skin eczema, and skin erosion.

\section{Materials and methods}

\subsection{Subjects}

A total of 42 infants (73 external ears) with auricle deformities from April 2018 to October 2018 identified by Guangzhou Children and Women's medical center's otolaryngologists were included in this study. Their mean ages at the initiation of ear splint therapy is $37.87 \pm 19.44$ days and the mean treatment duration is $47.21 \pm 17.36$ days. The types of auricle deformities include helix (11 cases), lop (5 cases), cupped (4 cases), prominent (6 cases), cryptotia (3 cases), stahl (3 cases), and mixed deformities (10 cases). The inclusion criteria were infants with clinical diagnosis of auricle deformities and without natural correction after 1-week follow-up observation, ${ }^{5}$ and their age below 3 months. Exclusive criteria are: (1) babies older than 3 months at the time of initiation of therapy; (2) skin concerns around the ear that need to be treated; (3) ear malformations characterized by a partial absence of the skin or cartilage requiring surgical correction; and (4) a history of allergic reaction to an adhesive tape. All patients' guardians were well informed and signed the consent form. In addition, the parents received modularized nursing care education when they were back to home to decrease the complications and obtain better clinical effect. The nursing care education included four parts: adhesive awareness, washing, redness alert, and cradle maintenance. The nurse in charge will implement the parents' education after the treatment.

\subsection{Correction materials}

The EarWell Infant Ear Correction System employs a simple structured method involving a cradle with a cup, a small rigid retractor, and a soft conchal former. The correction materials utilize polyurethane thermoplastic elastomer materials (TPE) without irritation, sanitation and toxicity to babies' skin. Recent studies have emphasized the timely application of modeling techniques since the better clinical outcome and less complications.

\subsection{Correction methods}

The EarWell Infant Ear Correction System is applied as follows:

(1) This readymade device comes in small, medium, and large sizes that can be adjusted according to patients' auricle shape.

(2) Skin preparation: Use disposable clipper to remove $3 \mathrm{~cm}$ of hair around the ear. 
(3) Skin degreasing: Degreasing the skin where the cradle, cup, and tape are adhered.

(4) The babies were held in their guardian's arms in a lateral decubitus position with the ear to be treated facing up.

(5) Apply the cradle, retractor, and conchal former in place. The average therapeutic time-span lasts over 4 weeks and return visit at weekly intervals. At every return visit, correction of auricle deformities was checked and the device adjusted until satisfactory correction was achieved, then continued to wear the device for another 7 days. If treatment is still needed, utilize tape to achieve immobilization.

(6) Patient education: Teach nursing methods for patients' guardians, such as modeling device cannot be wet. Prepare a tape for guardians if the device needs to reinforce. If the skin color has changed, the parents need to contact otolaryngologist for help. Photographs should be taken and bring at every visit.

\subsection{Parents' modular nursing care education}

(1) Adhesive: Instruct the parents that EarWell is affixed to baby's skin by a medical adhesive designed to maximize adhesion and minimize trauma. It will hold the EarWell reliably in place for approximately 14-17 days. Parents should inspect the cradle daily to verify its continued adhesive skin bond. When parents notice that the cradle adhesive begins to separate from the skin, call the nurse in charge to schedule an appointment, and ENT physician will adjust or reapply the EarWell cradles. Before the adjustment or re-applying procedure, parents should use the provided roll of tape to temporarily secure the EarWell to the skin. Cut a small piece of tape from the roll (3 inches or less) and apply over the top of the EarWell and then onto the skin of the baby. If the area of separation is larger than the width of the tape, use additional pieces of tape to secure it to the skin. There are also occasions when one or both EarWell cradles prematurely separate from the skin. This can occur when a child becomes unusually active or restless resulting in vigorous movement of arms and shoulders. Upon observing such separation, use the tape to secure the cradle to the skin in the manner described above. If the EarWell becomes completely dislodged, or for more than $50 \%$ of the EarWell surface area, do not attempt to tape it back in place. Immediately call the nurse in charge to schedule an appointment with ENT physician. It is important to note that the EarWell becoming temporarily dislodged will not affect the results or the duration of the therapy. Simply schedule an appointment with the nurse in charge and ENT physician as soon as possible.

(2) Washing: Because adhesives are an intricate part of the EarWell attachment, it should remain as dry as possible. It is recommended that parents do not wet the EarWell or attempt to wash it.

(3) Redness: EarWell is made of a clear material to permit ear visibility. If the ear becomes unusually red in color, report it to the nurse in charge and ENT physician.

(4) Cradle maintenance: Care should be given to keeping the cradle interior clear of milk, formula, or vomit. If any of these fluids penetrate to the inside of the cradle, make an appointment with charge nurse and ENT physician for the removal of the top shell and cleaning of the ear and EarWell components. More attention should be paid to protect the cradle interior from the presence of any vomit.

The patient modular nursing care education session will be complete by the charge nurse with theory teaching and skill demonstration session.

\subsection{Analysis of effectiveness of EarWell infant of ear correction system}

The severity of auricle deformity was evaluated preand post-treatment by both physician and guardian with modularized nursing care education, using the same auricle deformities visual analogue scales (VAS) rating system. We separately compared the physician's and guardian's rating pre- and post-therapy. The distinguish of physician and guardian's rating was also completed. Side effects and complications during therapy were investigated. Patient satisfaction was evaluated using Global esthetic improvement scale (GAIS). ${ }^{6}$ The satisfaction level is divided into exceptional improvement (excellent corrective result), very improved (marked improvement of the appearance, but not completely optimal), improved (improvement of the appearance, better compared with the initial condition, but a touchup is advised), unaltered (the appearance substantially remains the same compared with the original condition), and worsened (the appearance has worsened compared with the original condition). Total satisfaction was equal to the sum of exceptional improvement, very improved patient, and improved patient. 


\subsection{Statistical analysis}

Statistical analysis was undertaken using SPSS version 21.0, categorical data are expressed as percentages and examples. A chi-squared $\left(\mathrm{c}^{2}\right)$ statistic was used to evaluate a comparison among groups. Continuous data are expressed as "M \pm SD", $T$-test was applied to evaluate the comparison among groups if data were consistent with the Gaussian distribution and nonparametric tests applied to evaluate the comparison among groups if data were not in normal distribution. A value of $P \leq 0.05$ was considered statistically significant.

\section{Results}

\subsection{Analysis of effectiveness of EarWell Infant Ear Correction System with guardians' modularized nursing care education}

In the auricle deformities VAS rating system, where point 0-2 denoted severe deformity, 3-5 denoted moderate deformity, 6-8 denoted mild deformity, and 9-10 denoted normal. The mean initial pediatric otolaryngologist's rating was $6.51 \pm 0.84$, severe deformity auricle $(<4,16.43 \%)$, moderate deformity auricle $(4-7,78.08 \%)$, mild deformity or normal auricle $(>7,5.48 \%)$; the mean final pediatric otolaryngologist 'rating after completion of the treatment was $8.33 \pm 1.27$, severe deformity auricle $(<4,1.37 \%)$, moderate deformity auricle $(4-7,45.21 \%)$, mild deformity or normal auricle $(>7,53.42 \%)$, and normal auricle occupied $43.90 \%$. The comparison of the mean initial and final pediatric otolaryngologist's ratings for the auricle deformity showed significant improvement with EarWell Infant Ear Correction System combined with proper parents 'nursing education program'. The mean initial guardian's rating was $5.77 \pm 1.59$ and the mean finial guardian's rating after completion of the therapy was $8.19 \pm 2.38$. The comparison of the mean initial and final guardian's rating for the auricle deformity showed significant improvement with EarWell Infant Ear Correction System. The pediatric otolaryngologist's and guardian's rating is without significant difference (Table 1 and Figure 1).

\subsection{Complications of EarWell Infant Ear Correction System}

In our study, only a few and minor complications of auricular correction with EarWell Infant Ear Correction System were seen, such as eczema, skin irritation or erosion, and so on. All of the 42 patients completed the therapy without severe complications such as necrosis

\begin{tabular}{|c|c|c|c|c|}
\hline \multirow[t]{2}{*}{ Types } & \multicolumn{3}{|c|}{ Pediatric otolaryngologist' rating } & \multirow{2}{*}{$\begin{array}{l}\text { Guardian's } \\
\text { rating }\end{array}$} \\
\hline & Mean value & Deformity rating & Cases $n(\%)$ & \\
\hline & & $<4$ & $12(16.43)$ & \\
\hline \multirow[t]{3}{*}{ Initial rating } & $6.51 \pm 0.84$ & $4-7$ & $57(78.08)$ & $5.77 \pm 1.59$ \\
\hline & & $>7$ & $4(5.48)$ & \\
\hline & & $<4$ & $1(1.37)^{\star}$ & \\
\hline \multirow[t]{2}{*}{ Final rating } & $8.33 \pm 1.27^{\star}$ & $4-7$ & $33(45.21)$ & $8.19 \pm 2.38^{*}$ \\
\hline & & $>7$ & $39(53.42)^{*}$ & \\
\hline
\end{tabular}

Note: *represent significant difference for comparisons between initial and finial rating after completing the therapy, $P<0.05$. There is no missing data since the regular return visit of the patient is $100 \%$.

Table 1. Rating results comparison of the auricle deformity before and after the therapy

of the skin or cartilage. Six (14.63\%) patients showed repetitive scratching of the splinted ear, especially for those with older age. Three (7.14\%) patients developed eczema, and two (4.76\%) patients had mild skin erosion, which manifested the modularized parents' nursing care education will decrease the complication and engaged the parents during the therapy process (Table 2).

\subsection{Patients guardian's satisfaction analysis}

Patient satisfaction was evaluated by using GAIS, where it is divided into exceptional improvement, very improved, improved, unaltered, and worsened. Total satisfaction was equal to the sum of exceptional improvement, very improved patient, and improved patient. Compared with the therapy initiated, GAIS rate is only $26.19 \%$. Upon the completion of the treatment, the rate increases to $90.48 \%$, with statistically significant differences $(P<0.05)$ (Table 3$)$.

\section{Discussion}

Morphological abnormalities of the auricle are caused by abnormal positioning in the uterus as early as 9 weeks of pregnancy, or during and after delivery such as improper sleeping posture, malposition, and obstetric canal extrusion. ${ }^{7,8}$ Auricle morphological abnormalities include lop ear, stahl's ear, helical rim abnormality, constricted ear, cup ear, prominent ear, cryptotia, conchal crus, mixed ear deformity, preoperational unfolding, or post-operation fixed. The most common morphological abnormalities are prominent ear combined with cup ear. Although there is no life-threatening risk for patient with auricle deformities, patient with auricle deformities tend to suffer psychological distress manifesting as anxiety, low self-esteem, and behavioral problems. Earlier molding technique has been shown to be effective for correcting auricle deformities during the neonatal period. The advantage of early molding technique over 
A

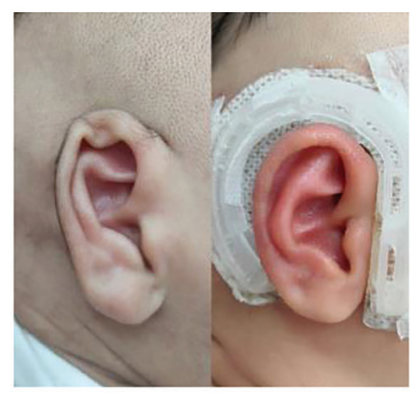

B

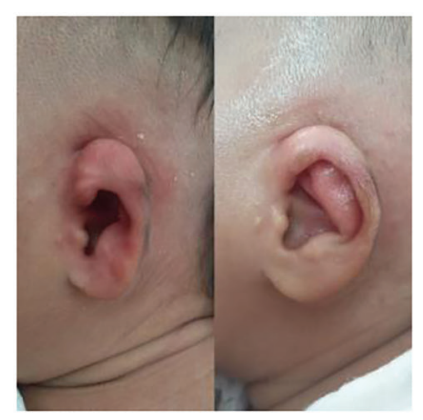

C

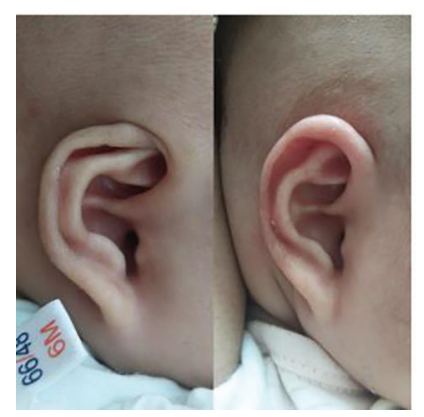

D

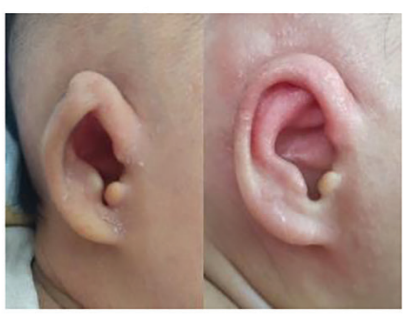

E

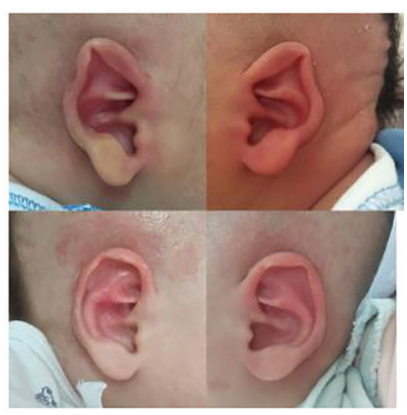

$\mathrm{F}$

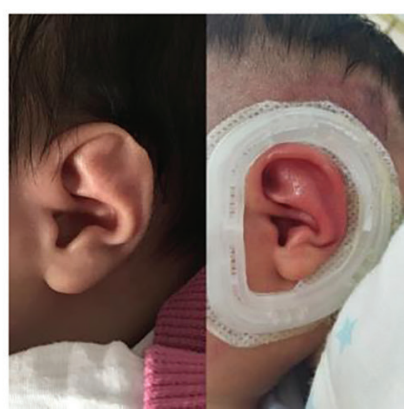

A. Born 30 days and after wearing for 17 days.

B. Born 3 days and after wearing for 13 days.

C. Born 32 days and after wearing for 28 days.

D. Born 4 days and after wearing for 22 days.

E. Born 8 day and after wearing for 55 days.

F. Born 1 day and after wearing for 16 days.

Note: These pictures list before treatment (left in each group) and after treatment (right in each group).

Figure 1. Treatment example of EarWell Infant Ear Correction System.

\begin{tabular}{lcc}
\hline Complication types & Cases $n$ & Percentage (\%) \\
\hline \hline Scratching ears & 6 & 14.29 \\
Skin eczema & 3 & 7.14 \\
Skin erosion & 2 & 4.76 \\
\hline
\end{tabular}

Table 2. Complications observed during therapy.

otoplasty include fewer complications and lower potential for psychological distress. ${ }^{9}$

Regarding the auricle deformities self-correction concern, controversy may exist regarding the possibility of unnecessary treatment. However, it is still impossible to predict which ears will spontaneously correct itself, and if the deformities cannot be self-resolved, the patient has to undergo an operation for the deformities after 6 years of age or auricle development around $90 \%$ of adult size. ${ }^{10}$

Otoplasty is the traditional auricle deformity surgical correction treatment with unpredictable operation times and length of hospital stay; the risk of complications of surgical correction is commonly underestimated, there is around a $5 \%$ risk of early complication (hematoma, infection, fistulae caused by stitches and granulomas, 


\begin{tabular}{lccccc}
\hline Types & $\begin{array}{c}\text { Exceptional } \\
\text { improvement }\end{array}$ & Very improved & Improved & Unaltered & $\begin{array}{c}\text { Worsened } \\
\text { satisfaction } n \text { (\%) }\end{array}$ \\
\hline \hline Pre-therapy & 0 & 2 & 9 & 31 & 0 \\
Post-therapy & 5 & 14 & 19 & 4 & 0 \\
\hline
\end{tabular}

Note: *represents significant difference for comparisons between pre-therapy and post-therapy rating, $P<0.05$.

Table 3. Rating results comparison of the patients' guardians satisfaction before and after the therapy.

allergic reactions, pressure ulcers, feelings of pain, and asymmetry in side comparison) and a $20 \%$ risk of late complications (recurrences, telephone ear, excessive edge formation, auricle fitting too closely, narrowing of the auditory canal, keloids, and complete collapse of the ear). ${ }^{11,12}$

The first medical publication on nonsurgical correction of congenital auricular deformities was published in the late 1980s. However, there are still many unanswered questions regarding the usage of ear splints. It is evident that splinting (include EarWell Infant Ear Correction System) is an elegant method to correct auricle deformities in the newborn. The external ear is easily pliable due to the flexibility of the cartilage. The high levels of estrogen received from the mother is one cause of flexibility of the cartilage. The pliability of cartilage depends on the composition of the extra-cellular matrix and especially on the amount of hyaluronic acid. ${ }^{2}$ Taking advantage of the temporary malleability of the infant ear cartilage, the EarWell Infant Ear Correction System can apply a combination of anterior and posterior forces that selectively shape and expand target ears. ${ }^{13}$ Previously, ear molding has required the use of various molds, feeding tubes, surgical tapes, and splints, which are cumbersome for guardians and difficult to keep in position. The EarWell Infant Ear Correction System is an approved adhesive appliance that attaches to the child's skin to mold the different kinds of auricle deformities into a normal anatomical shape, and it is convenient for operation, cost-friendly, and with less complications. ${ }^{7}$ Recent study revealed that use of this device can lead to significant corrective clinical outcome with better esthetic detail than what was achieved with previous molding techniques or through surgical intervention. ${ }^{14}$ While EarWell Infant Ear Correction System has been successfully used to correct auricle deformities in the newborns, and its unique parents' modularized nursing care education session enhance the clinical outcome through decreasing the complications. There is no comprehensive evidence on the length of time needed for splinting, majority of the clinical practice adjust the duration of treatment according to the patients' specific condition during follow-up visit. Many EarWell Journal articles are published and clinical studies were performed, which represent clinical breakthrough in how to treat auricle deformities. Through more clinical practice and studies with EarWell, caregivers and parents with well-educated daily nursing skills may have an opportunity to make a positive difference in the lives of children, helping to decrease the psychological stress and eliminating the need for invasive surgical correction later in life with increased socioeconomic cost.

In our study, the mean pre-therapy pediatric otolaryngologist's auricle deformities VAS rating was $6.51 \pm 0.84$ and the mean post-therapy rating was $8.33 \pm 1.27$. The difference between pre- and post-therapy was statistically significant $(P<0.05)$, as measured by quantitative rating scales, and demonstrates the EarWell Infant Ear Correction System significantly improved auricle deformities in babies. The mean pre-therapy patient' guardians auricle deformities VAS rating was $5.77 \pm 1.59$ and the mean post-therapy rating was $8.19 \pm 2.38$. The difference between pre- and post-therapy was statistically significant $(P<0.05)$, and this also indicates EarWell significantly improved the patient's auricle deformities. No significant difference was found between the pediatric otolaryngologists and patient 'guardians' ratings on completion of the therapy. The complications of EarWell Infant Ear Correction System have been minor and small with adequate parents modularized nursing care education and daily practice, 2 patients $(4.76 \%)$ had mild skin erosion, 3 patients $(7.14 \%)$ had skin eczema, and 6 patients $(14.29 \%)$ scratched ears in total 42 patients (73 external ears). Before EarWell therapy and at the end of the therapy, there is an obvious GAIS satisfaction rating improved from 26.19 to $90.48 \%$, a $P<0.05$ was considered statistically significant, which indicates that the modularized parents' nursing care session provides parents with a complete engagement opportunity and creates a real patient-centered treatment environment.

The EarWell Infant Ear Correction System with its unique modularized parents' nursing care education session was shown to be effective and safe in eliminating or reducing the need of surgery in auricle deformities with minor complications and reasonable clinical outcome. 


\section{Ethical approval}

Ethical issues are not involved in this paper.

\section{References}

1. Su Y, Ding ZW, Ma JL. Deformational auricular abnormalizes in newborn infants: Early recognition and non-surgical correction. Chin $\mathrm{J}$ Otol. 2018;16:38-41 (in Chinese).

2. Zhou X, Wang $Y$, Zhang QG, et al. Selection and application of ear reconstruction surgery for Chinese microtia. Chin J Otorhinolaryngol Head Neck Surg. 2015;50:192-196 (in Chinese).

3. Staindl O, Siedek V. Complications of auricular correction. GMS Curr Top Otorhinolaryngol Head Neck Surg. 2007;6:Doc 03.

4. Lee Y, Kim YS, Lee WJ, et al. Proposal of a classification system for the assessment and treatment of prominent ear deformity. Aesthetic Plast Surg. 2018;42:759-765.

5. Paul-Dauphin A, Guillemin F, Virion JM, Briancon S. Bias and precision in visual analogue scales: A randomized controlled trail. Am J Epidemiol. 1999;150:1117-1127.

6. Haytoğlu S, Haytoğlu TG, Kuran G, Yıldırım I, Arıkan OK. Effects of cartilage scoring in correction of prominent ear with incisionless otoplasty technique in pediatric patients. J Int Adv Otol. 2017;13:21-27.

7. Zhou X, Liu T, Wang Y, et al. The methods of total ear reconstruction for microtia: Selection and application. Chin J Plast Surg. 2017;33:34-39 (in Chinese).

8. Xing WS, Qiang J, Hu JT, Wang Y, Wang BQ, Zhang QG. The application of 2D printing of rib

\section{Conflicts of interest}

All contributing authors declare no conflicts of interest.

cartilage in personalized ear framework fabrication. Chin J Plast Surg. 2018;34:206-209 (in Chinese).

9. Jiang DW, Geng J, Li H, Hu XY. Microtia of I degree retroauricular flap combined with autologous rib cartilage primary transplantation of auricular reconstruction for correcting and repairing microtia of I degree. Prac J Med Pharm. 2016;33:401-403 (in Chinese).

10. Zhang LC, Chen Y, Zhang TY. Three-stage surgery of combining auricle reconstruction, meatoplasty and tympanoplasty for patients with congenital microtia-atresia. Chin J Otorhinolaryngol Head Neck Surg. 2015;50:197-202 (in Chinese).

11. Ma FL, Meng BX, Wang L, Jian YL, Wang CW. To explore the methods and effects of simultaneous surgical correction of ear malformation and mild hemifacial microsomia. Henan J Surg. 2018;24: 25-26 (in Chinese).

12. Daniali LN, Rezzadeh K, Shell C, et al. Classification of newborn ear malformations and their treatment with the EarWell infant ear correction system. Plast Reconstr Surg. 2017;139:681-691.

13. Cao J. Construction of neonatal ear morphology database and analysis of related factors of auricular deformity. Med Inf. 2015;28:262-263 (in Chinese).

14. Zou F, Li Q, Zhuang QE, Zhang Y. A study of ear molding in newborns and infants with auricle deformities. Chin J Otorhinolaryngol Integ Med. 2017;25:355-358 (in Chinese). 